\title{
Serological evaluation of antigen Tp0693 for diagnosis of syphilis
}

\author{
LI ZHANG ${ }^{1,2^{*}}$, MEIXIA DENG ${ }^{2 *}$, XIAOHONG ZHANG ${ }^{2}$, WEIGUO YIN ${ }^{3,4}$, \\ WEN LIU $^{2}, \mathrm{XI} \mathrm{LUO}^{2}$, JIE YANG $^{5}$, FEI CHEN ${ }^{5}$ and FEIJUN ZHAO ${ }^{2}$
}

\author{
${ }^{1}$ Department of Laboratory, The First People's Hospital of Changde Affiliated to The University of South \\ China, Changde, Hunan 415000; ${ }^{2}$ Hunan Provincial Key Laboratory for Special Pathogens Prevention and \\ Control, Institute of Pathogenic Biology, University of South China, Hengyang, Hunan 421001; ${ }^{3}$ Department \\ of Laboratory, Yuebei People's Hospital, Shaoguan, Guangdong 512026; ${ }^{4}$ Department of Molecular Biology \\ Research Center, Qingyuan People's Hospital, Qingyuan, Guangdong 511500; ${ }^{5}$ Department of Clinical \\ Medicine, Medical College, University of South China, Hengyang, Hunan 421001, P.R. China
}

Received November 7, 2016; Accepted June 15, 2017

DOI: $10.3892 /$ etm.2017.5158

\begin{abstract}
The aim of the present study was to evaluate the diagnostic value of the Treponema pallidum (Tp) antigen Tp0693 for syphilis. ELISA was used to examine the serum levels of Tp0693. The sample-to-cutoff ratio (S/CO) value was used to generate a receiver operating characteristic (ROC) curve. A correlation analysis was performed to compare the detection efficacy of Tp0693-specific ELISA, Treponema pallidum Particle Agglutination (TPPA), Tolulized Red Unheated Serum test (TRUST) and LiZhu ${ }^{\mathrm{TM}}$ Tp-ELISA. The area under the ROC curve was 0.99 , indicating good diagnostic efficacy. When the diagnostic specificity reached $100 \%$, the diagnostic sensitivity was up to $93.5 \%$. Tp0693-specific ELISA results were not correlated with those of TPPA, TRUST and LiZhu ${ }^{\mathrm{TM}}$ Tp-ELISA (correlation coefficient, 0.122, 0.114 and 0.025 , respectively). The latent syphilis rate was highest $(12 \%, 9 / 75)$ for all syphilis specimens with a S/CO in the grey area. In conclusion, for syphilis specimens with a S/CO in the grey area, TPPA should be used for further confirmation of the diagnosis. Tp0693 may be used as a diagnostic antigen for syphilis; however, further study regarding its potential use is required.
\end{abstract}

Correspondence to: Professor Feijun Zhao, Hunan Provincial Key Laboratory for Special Pathogens Prevention and Control, Institute of Pathogenic Biology, University of South China, 28 Changsheng West Road, Hengyang, Hunan 421001, P.R. China

E-mail: nhdxzhfj@163.com

*Contributed equally

Key words: Treponema pallidum, Tp0693, secretion protein, serological diagnosis

\section{Introduction}

Syphilis is a sexually transmitted disease caused by Treponema pallidum (Tp), which mainly attacks genital and mucous membranes in the early stage and affects all systems in the advanced stage (1). Based on the disease course and symptoms, syphilis may be classified as primary, secondary, tertiary and latent syphilis (2). The prognosis of syphilis patients is good if the disease is detected early and subjected to standard treatment (3). The incidence of syphilis has been increasing worldwide $(4,5)$; for instance, it ranks first among all sexually transmitted diseases in China (6,7). Atypical symptoms or untreated latent syphilis may develop into serious cardiovascular syphilis and neurosyphilis and may finally be life-threatening (8).

At present, the clinical diagnosis of syphilis is based on a combination of the patient's personal history, clinical symptoms and laboratory tests, the latter of which are particularly important for patients with latent syphilis without any clinical symptoms. At present, the laboratory diagnosis of syphilis is mainly based on serological tests. The new syphilis algorithm is of high specificity and sensitivity (9), using Tp-specific antibody tests such as enzyme or chemiluminescent immunoassays as screening tests and the rapid plasma regain (RPR) test or the Tolulized Red Unheated Serum test (TRUST) for diagnosis as well as evaluation of treatment efficacy and relapse (10). When a patient with suspected Tp infection is false positive, specific antibody detection methods such as Tp Particle Agglutination (TPPA) or fluorescent treponemal antibody absorption are performed for confirmation (10). However, the sensitivity of Tp-specific antibody detection kits requires to be improved for early diagnosis of syphilis (11). TRUST and RPR are non-specific antibody detection assays with a high false-positive rate (12) and may not be suitable for evaluation of treatment efficacy due to prolonged observation time and serofast phenomenon (13), particularly in patients with low titers.

The syphilis-specific recombinant diagnostic antigens in $\mathrm{Tp}$ antibody detection kits are predominantly membrane lipoproteins such as chimera of $\mathrm{Tp} 17, \mathrm{Tp} 47, \mathrm{Tp} 15$ and 
treponemal membrane protein A $(14,15)$. Detection of syphilis using these antigens has certain disadvantages. For instance, Baughn et al (16) found that human fibronectin was in high homology with the 411PGTEYT426 sequence of the Tp47 antigen, as well as with antigens of Treponema endemicum and Treponema pertenue, leading to false positive reactions. Furthermore, the number and types of Tp outer membrane proteins are rare (17); therefore, it is necessary to identify novel syphilis-specific antigens.

$\mathrm{Tp} 0693$ is a hypothetical protein with unknown function (18). The preliminary results demonstrated that $\mathrm{Tp} 0693$ has strong immunogenicity and is specifically recognized in sera from patients with syphilis infection during the screening of antigens for syphilis. A bioinformatics analysis, including transmembrane, signaling peptide, subcellular localization and epitope analyses, indicated that it is a secretion protein with strong immunogenicity in the present study. Secretion proteins of other pathogens, such as leishmaniasis (19), tuberculosis (20), Fasciola (21) and gasterophilosis (22) have been found to serve as diagnostic antigens. However, whether Tp0693 is a diagnostic antigen for syphilis has remained elusive. The present study evaluated the serodiagnostic value of Tp0693 in syphilis at various stages, including early and latent syphilis.

\section{Materials and methods}

Strains and plasmids. Tp Nichols standard strains were preserved by inoculation in a total of 8 male New Zealand rabbits (3.0-3.5 kg; 240 days old; Department of Laboratory Animal, University of South China, Changde, China) and rabbits were housed at $18-20^{\circ} \mathrm{C}, 60-65 \%$ humidity with a $12 \mathrm{~h} \mathrm{light/dark} \mathrm{cycle} \mathrm{and} \mathrm{free} \mathrm{access} \mathrm{to} \mathrm{food} \mathrm{and} \mathrm{water.} \mathrm{All}$ animal experiments were approved by the Animal Welfare Committee of University of South China. The Escherichia (E.) coli strain JM109 (Invitrogen; Thermo Fisher Scientific, Inc., Waltham, MA, USA) was used for DNA cloning and BL21 (DE3; Merck KGaA, Darmstadt, Germany) was used for protein expression were provided by the Institute of Pathogenic Biology, University of South China (Chengyang, China).

Patients and samples. A total of 168 syphilis clinical serum samples were obtained from patients with clinically diagnosed syphilis at the First People's Hospital of Changde (Changde, China) from September 2014 to September 2015, including 36 cases of primary syphilis, 41 cases of secondary syphilis, 12 cases of tertiary syphilis, 75 cases of latent syphilis and 4 cases of congenital syphilis. A total of 153 cases without syphilis infection were used as controls, including 2 cases of epidemic hemorrhagic fever, 22 cases of Epstein-Barr (EB) virus infection, 10 cases salmonella infection, 29 cases of rheumatic disease, 1 case of multiple myeloma, 1 case of cytomegalovirus infection, 51 healthy patients who visited the hospital for physical examination and 27 pregnant women. All clinical information was obtained from the medical records of the subjects. Prior written informed consent was obtained from each subject. The study was approved by the ethics review board of the University of South China (Changde, China).
All specimens were stored at $-20^{\circ} \mathrm{C}$ prior to examination with a TPPA kit (VN40810; Fujirebio Diagnostics, Inc., Japan), a TRUST kit (2014082504; Shanghai Rongsheng Bio-Tech Co., Ltd., Shanghai, China) and a LiZhu ${ }^{\mathrm{TM}}$ Tp-ELISA kit (2014082601; Lvzhu Pharmaceutical Group Co., Ltd., Beijing, China).

Construction of pET30a-Tp0693 plasmid. The primers for the Tp0693 gene were 5'-CGCGGATCCATGGACCGTTTT TTTTGTACGG-3' and 5'-CCGCTCGAGTTACAGGAA GCACTGGAGC-3'. The Tp Nichols strain was used as a template for polymerase chain reaction PCR amplification of the Tp0693 gene. The Tp0693 gene was then cloned into a pET30a $(+)$ vector (Novagen; Merck KGaA) to construct the pET30a-Tp0693 plasmid. The pET30a-Tp0693 plasmid was identified by colony screening and sequencing.

Expression and purification of recombinant proteins. The recombinant plasmid pET30a-Tp0693 was transfected into E. coli BL21 (DE3), which was then cultured in lysogeny broth (Bioleaf Science, Inc., Shanghai, China) and centrifuged at $37^{\circ} \mathrm{C}$ with kanamycin at $80 \mathrm{x} \mathrm{g}$. Isopropyl $\beta$-D-1-thiogalactopyranoside (IPTG; Bioleaf Science, Inc.) at a final concentration of $0.5 \mathrm{mM}$ was added when the optical density (OD) reached $\sim 0.6$. The bacteria were then centrifuged at $30^{\circ} \mathrm{C}$ for $4 \mathrm{~h}$ with kanamycin at $50 \mathrm{x} \mathrm{g}$. Finally, the bacteria were lysed in a buffer containing $50 \mathrm{mM}$ Trs- $\mathrm{HCl}$ (pH 7.8), $300 \mathrm{mM} \mathrm{NaCl}, 10 \mathrm{mM}$ imidazole, $20 \%$ glycerol and $1.5 \%$ Triton X-100, centrifuged at $16,000 \mathrm{x} \mathrm{g}$ for $10 \mathrm{~min}$ and the supernatant was collected. The recombinant protein $\mathrm{Tp} 0693$ was purified by $\mathrm{Ni}^{2+}$-nitroloacetic acid affinity chromatography (Qiagen AB, Sollentuna, Sweden).

Western blot analysis. The purified recombinant protein Tp0693 (15 $\mu \mathrm{g}$, Lowory assay) was separated by $12 \%$ SDS-PAGE and then transferred onto a polyvinylidene difluoride membrane. After blocking with 5\% skimmed milk, the membrane was incubated overnight at $4^{\circ} \mathrm{C}$ with primary anti-polyhistidine tag (His)-labeled monoclonal antibody (1:1,000; 20150201; Auragene Bioscience Co.,Ltd.,Changsha, China) or with serum from syphilis-positive or -negative patients. After washing for 3 times, secondary horseradish peroxidase (HRP)-labeled goat anti-human immunoglobulin G (IgG) antibody (1:100; 20150312; Shanghai Yanhui Biology Technology Co., Ltd., Shanghai, China) was added, followed by incubation for $1 \mathrm{~h}$ at $37^{\circ} \mathrm{C}$. Finally, the membrane was developed with enhanced chemiluminescence plus reagent (Pierce; Thermo Fisher Scientific, Inc.). The developed film was scanned using the AlphaImager gel imaging system (G:BOX Chemi XXX9; Syngene, Cambridge, UK). The western blot images were analyzed using Quantity One software 3.0 (Bio-Rad Laboratories, Inc., Hercules, CA, USA).

Establishment of indirect Tp0693-ELISA method and TPPA, RPR and LiZhu ${ }^{T M}$ Tp-ELISA testing. The recombinant protein Tp0693 was diluted with carbonate buffer ( $\mathrm{pH}$ 9.6) to a concentration of $15 \mu \mathrm{g} / \mathrm{ml}$ and was used as the antigen. The Tp0693 solution was then added to 96 -well microtiter plates at $100 \mu 1$ per well and incubated overnight at $4^{\circ} \mathrm{C}$. The plate was 
washed 5 times with PBS containing Tween-20 (PBST), and was blocked by incubation with $5 \%$ skimmed milk overnight at $4^{\circ} \mathrm{C}$. Sample serum $(100 \mu \mathrm{l} ; 1: 100$ dilution) was added to each well and incubated for $60 \mathrm{~min}$ at $37^{\circ} \mathrm{C}$. After washing with PBST for 5 times, secondary antibody (1:1,000 dilution; HRP-labeled goat anti-human IgG) was added, followed by incubation at $37^{\circ} \mathrm{C}$ for $1 \mathrm{~h}$. The chromogenic agent was added and the absorbance was detected at $450 \mathrm{~nm}$ wavelength for obtaining sample-to-cutoff ratio $(\mathrm{S} / \mathrm{CO})$ values of sample serum. The experiment was repeated 3 times in parallel. All samples were then analyzed by TPPA, RPR and LiZhu ${ }^{\mathrm{TM}}$ Tp-ELISA according to the kit instructions.

Statistical analysis. Statistical analysis was performed using SPSS 18.0 software (SPSS, Inc., Chicago, IL, USA). A receiver operating characteristic (ROC) curve was plotted. The area under the curve (AUC) was identified, and the sensitivity and specificity were calculated. The S/CO values of Tp0693-ELISA and LiZhu ${ }^{\mathrm{TM}}$ Tp-ELISA were continuous variables, and titers of TPPA and TRUST were categorical variables. A Spearman correlation analysis was used. $\mathrm{P}<0.05$ was considered to indicate a statistically significant difference.

\section{Results}

Identification of engineered strain. To identify the engineered Tp0693-expressing strain, the recombinant plasmids were identified by PCR, restriction digestion and sequencing. The expected specific target band at 1,323 bp was visible, as presented in Fig. 1. The gene sequences were compared with the published gene sequences in GenBank (https://www.ncbi.nlm.nih.gov/genbank/) through the Basic Local Alignment Search Tool with $100 \%$ compatibility. This indicated that the recombinant Tp0693-expressing plasmid was successfully constructed.

Expression, purification and identification of recombinant proteins. To determine the expression, purification and identification of recombinant proteins, the diluted anti-His antibody, as well as serum from patients with or without syphilis were used as the primary antibodies or negative control for western blot analysis. Tp0693 expression was induced by IPTG. The identification of the $\mathrm{Ni}^{2+}$ column-purified product was examined by SDS-PAGE, as presented in Fig. 2A. The His antibody-reactive target band with a molecular weight of $\sim 52 \mathrm{kDa}$ was slightly larger than the standard value (47.66 kDa) (18), possibly due to the His tag at the $\mathrm{N}$ terminus. As presented in Fig. 2B, no immunoreactivity was observed with the serum from subjects without syphilis, while immunoreactive bands were obtained with anti-His antibody and serum from patients with syphilis. This indicated that after the expression, purification and western blot identification, the target protein was suitable for use in the subsequent ELISA experiments.

ROC curve analysis. The S/CO of the Tp0693-specific ELISA and clinical diagnosis of syphilis were used to draw a ROC curve, which is presented in Fig. 3. The AUC was 0.990. When the $\mathrm{S} / \mathrm{CO}$ was $<1.368$, the sensitivity was $100 \%$ and when the $\mathrm{S} / \mathrm{CO}$ was $>2.721$, the specificity was $100 \%$. This indicated that Tp0693 had a good diagnostic value in syphilis screening, and is expected to become a novel antigen candidate for the diagnosis of syphilis.

Comparison of TPPA, TRUST, LiZhuTM Tp-ELISA and Tp0693-ELISA. To determine the diagnostic efficacy of TPPA, TRUST, LiZhu ${ }^{\mathrm{TM}}$ Tp-ELISA and Tp0693-ELISA, the ROC curve was analyzed. The S/CO value of 2.721 in Tp0693-ELISAwas used as a cut-off value. As presented in Tables I and II, the detection sensitivity of the Tp0693-ELISA method was $93.5 \%$, which was higher than that of TRUST (79.2\%). The detection sensitivity of Tp0693-ELISA was $97.2 \%$ for primary syphilis, $100 \%$ for secondary syphilis, $91.7 \%$ for tertiary syphilis, $88 \%$ for latent syphilis and $100 \%$ for congenital syphilis. The distribution of the S/CO values for Tp0693-ELISA is presented in Fig. 4, demonstrating that the mean $\mathrm{S} / \mathrm{CO}$ value for serum samples from patients with $\mathrm{Tp}$ infection was higher than that of serum from patients without syphilis. This result indicated that analysis of Tp0693 may be used to specifically identify syphilis-infected sera.

Correlation analysis of TPPA, TRUST, LiZhu ${ }^{T M}$ Tp-ELISA and Tp0693-ELISA. To determine the correlation of TPPA, TRUST, ELISA and Tp0693-ELISA, a Spearman correlation analysis was performed. The correlation coefficient was $0.122(\mathrm{P}>0.05)$ for the Tp0693-ELISA S/CO value and the TPPA titer, 0.114 ( $P>0.05)$ for the Tp0693-ELISA S/CO value and the TRUST titer, and $0.025(\mathrm{P}>0.05)$ for the Tp0693-ELISA S/CO value and the LiZhu ${ }^{\mathrm{TM}} \mathrm{Tp}$ ELISA OD value. As presented in Fig. 5, the S/CO value of the Tp0693-ELISA was not increased with the TPPA titer, TRUST titer or LiZhu ${ }^{\mathrm{TM}} \mathrm{Tp}$-ELISA OD value. These results indicated that the S/CO value of Tp0693-ELISA was not correlated with TPPA or TRUST titers, or the LiZhu ${ }^{\mathrm{TM}}$ Tp-ELISA OD value.

Grey area analysis. To analyze the grey area of the Tp0693-ELISA S/CO value, its distribution was analyzed. The grey area of the Tp0693-ELISA results was identified to be between 1.368 (highest $\mathrm{S} / \mathrm{CO}$ when the sensitivity was $100 \%$ ) and 2.721 (the lowest S/CO when the specificity was $100 \%)$. The grey area distribution is presented in Table III, and it was highest (14.8\%) among patients with latent syphilis. In the cross-reaction examination, typhoid fever $(80 \%)$ and rheumatic diseases $(55.2 \%)$ ranked highest. As presented in Fig. 6, in the grey zone of syphilis-positive samples, the TPPA titers were all $\geq 1: 80$, part of the TRUST results were negative, and the OD values of the LiZhu ${ }^{\mathrm{TM}} \mathrm{Tp}$-ELISA were all $>0.15$. In the syphilis-negative specimens, TPPA and TRUST results were all negative; however, one sample was positive according to $\mathrm{LiZhu}^{\mathrm{TM}} \mathrm{Tp}$-ELISA. This result indicated that Tp0693 is a novel syphilis-specific antigen, whose diagnostic value may be limited, and further study is required to explore its associated functions.

\section{Discussion}

At present, the antigen screening targets for syphilis diagnosis are Tp membrane lipoprotein $(14,15,23,24)$ or flagellin $(25)$; however, they have certain limitations. The number and expression rate of syphilis-specific plasma membrane lipoproteins 
Table I. Sensitivity of TRUST, TPPA, Tp0693-ELISA and LiZhu ${ }^{\mathrm{TM}}$ Tp-ELISA in the detection of different stages of syphilis.

\begin{tabular}{|c|c|c|c|c|c|c|c|c|c|c|c|c|}
\hline \multirow[b]{2}{*}{$\begin{array}{l}\text { Sample } \\
\text { type }\end{array}$} & \multicolumn{3}{|c|}{ Tp0693-ELISA } & \multicolumn{3}{|c|}{ LiZhu $^{\mathrm{TM}}$ Tp-ELISA } & \multicolumn{3}{|c|}{ TPPA } & \multicolumn{3}{|c|}{ TRUST } \\
\hline & Positive & Negative & $\begin{array}{c}\text { Sensitivity } \\
(\%)\end{array}$ & Positive & Negative & $\begin{array}{c}\text { Sensitivity } \\
(\%)\end{array}$ & Positive & Negative & $\begin{array}{c}\text { Sensitivity } \\
(\%)\end{array}$ & Positive & Negative & $\begin{array}{c}\text { Sensitivity } \\
(\%)\end{array}$ \\
\hline PS & 35 & 1 & 97.2 & 36 & 0 & 100 & 36 & 0 & 100 & 24 & 12 & 66.7 \\
\hline SS & 41 & 0 & 100 & 41 & 0 & 100 & 41 & 0 & 100 & 37 & 4 & 90.2 \\
\hline $\mathrm{TS}$ & 11 & 1 & 91.7 & 12 & 0 & 100 & 12 & 0 & 100 & 12 & 0 & 100 \\
\hline LS & 66 & 9 & 88 & 75 & 0 & 100 & 75 & 0 & 100 & 56 & 19 & 74.7 \\
\hline CS & 4 & 0 & 100 & 4 & 0 & 100 & 4 & 0 & 100 & 4 & 0 & 100 \\
\hline AS & 157 & 11 & 93.5 & 168 & 0 & 100 & 168 & 0 & 100 & 152 & 16 & 79.2 \\
\hline
\end{tabular}

The S/CO value of 2.721 in Tp0693-ELISA that maximizes expression sensitivity + specificity - 1 was used as a cut-off value. PS, primary syphilis; SS, secondary syphilis; TS, tertiary syphilis; LS, latent syphilis; CS, congenital syphilis; AS, all syphilis samples; TPPA, Tp Particle Agglutination; TRUST, Tolulized Red Unheated Serum test; Tp, Treponema pallidum.

A

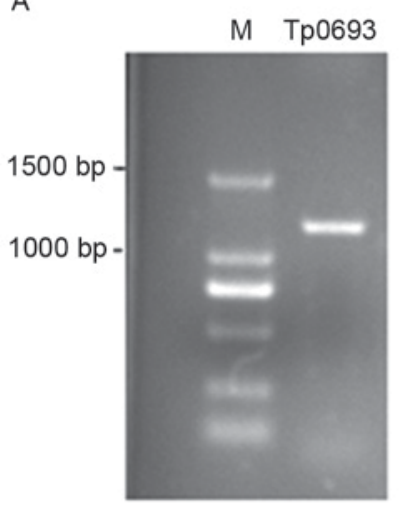

B

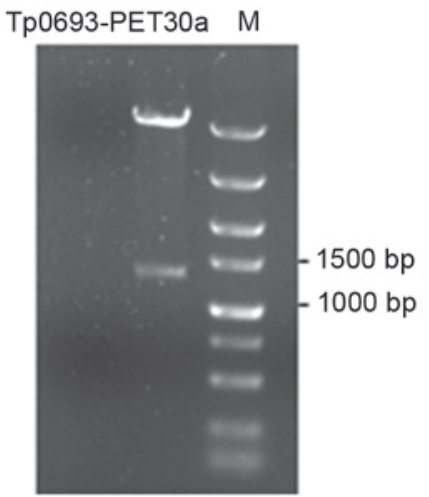

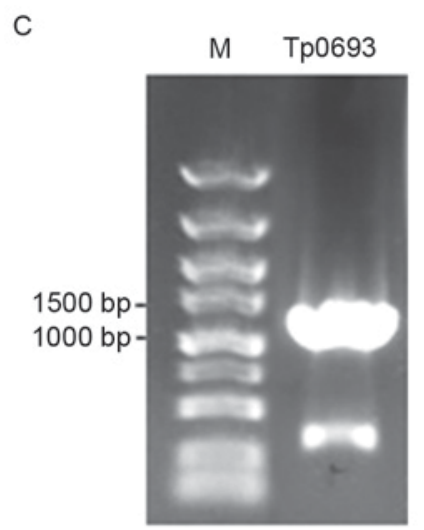

Figure 1. Amplification and identification of the Tp0693 gene. (A) PCR products of TP0693 from the genome of the Treponema pallidum Nichols strain. (B) Recombinant plasmid pET30a(+)/Tp0693 digested with BamHI and XhoI. (C) PCR products of TP0693 from recombinant expression in bacteria. PCR, polymerase chain reaction.

A

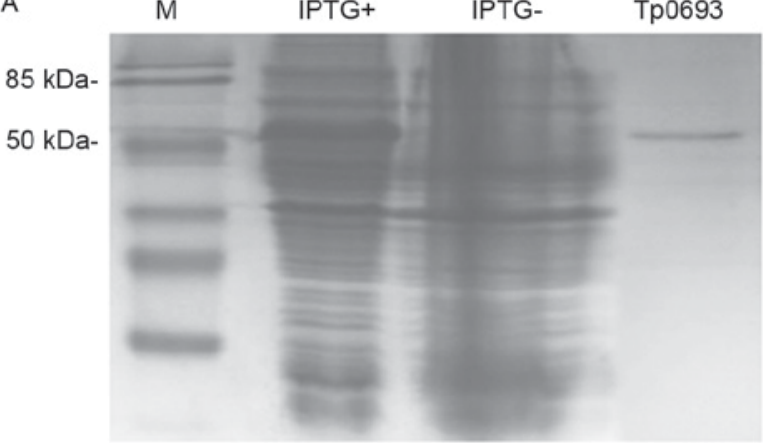

B

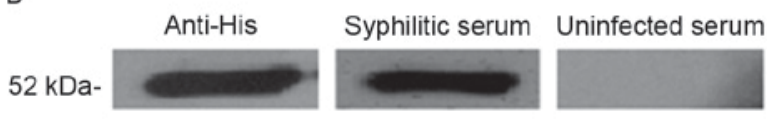

Figure 2. Expression, purification and identification of recombinant protein Tp0693. (A) SDS-PAGE analysis of the expression of Tp0693 induced with or without IPTG and the purified Tp0693. (B) Western blot analysis of Tp0693 with anti-His monoclonal antibody, syphilitic serum containing antibodies and uninfected serum. (+/-) IPTG, with/without isopropyl $\beta$-D-1-thiogalactopyranoside; His, polyhistidine tag.

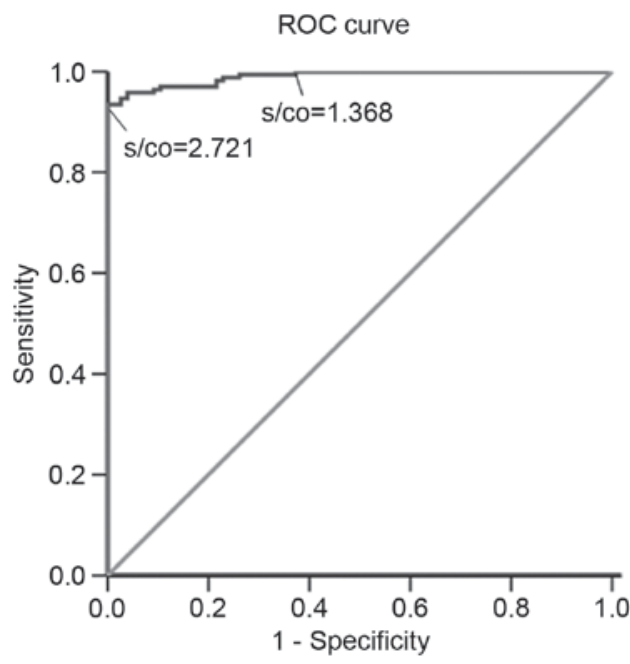

Figure 3. ROC curve for Tp0693-specific ELISA. 1-specificity (true negative rate) is displayed on the $\mathrm{x}$-axis and the sensitivity (true positive rate) is displayed on the y-axis. The AUC of the ROC curve was between 0.5 and 1.0. For AUC $>0.5$, an AUC closer to 1 is indicative of a better diagnostic efficacy. The AUC is sensitive between 0.7 and 0.9 , and has a high sensitivity when it is $>0.9$. In the present study, the AUC was 0.990. ROC, receiver operating characteristic; AUC, area under curve; S/CO, sample-to-cutoff ratio. 
Table II. Specificity of TRUST, TPPA, Tp0693-ELISA and LiZhu ${ }^{\mathrm{TM}}$ Tp-ELISA in the detection of sera from uninfected controls and potentially cross-reactive infections.

\begin{tabular}{|c|c|c|c|c|c|c|c|c|c|c|c|c|}
\hline \multirow[b]{2}{*}{$\begin{array}{l}\text { Sample } \\
\text { type }\end{array}$} & \multicolumn{3}{|c|}{ Tp0693-ELISA } & \multicolumn{3}{|c|}{ LiZhu $^{\mathrm{TM}}$ Tp-ELISA } & \multicolumn{3}{|c|}{ TPPA } & \multicolumn{3}{|c|}{ TRUST } \\
\hline & Positive & Negative & $\begin{array}{c}\text { Specificity } \\
(\%)\end{array}$ & Positive & Negative & $\begin{array}{c}\text { Specificity } \\
(\%)\end{array}$ & Positive & Negative & $\begin{array}{c}\text { Specificity } \\
(\%)\end{array}$ & Positive & Negative & $\begin{array}{c}\text { Specificity } \\
(\%)\end{array}$ \\
\hline $\mathrm{PM}$ & 0 & 37 & 100 & 5 & 32 & 86.5 & 0 & 37 & 100 & 0 & 37 & 100 \\
\hline $\mathrm{PE}$ & 0 & 51 & 100 & 12 & 39 & 76.5 & 0 & 51 & 100 & 0 & 51 & 100 \\
\hline EBV & 0 & 22 & 100 & 0 & 22 & 100 & 0 & 22 & 100 & 0 & 22 & 100 \\
\hline Sty & 0 & 10 & 100 & 0 & 10 & 100 & 0 & 10 & 100 & 0 & 10 & 100 \\
\hline $\mathrm{RD}$ & 0 & 29 & 100 & 0 & 2 & 100 & 0 & 29 & 100 & 0 & 29 & 100 \\
\hline EHF & 0 & 2 & 100 & 0 & 2 & 100 & 0 & 2 & 100 & 0 & 2 & 100 \\
\hline AN & 0 & 151 & 100 & 15 & 136 & 88.9 & 0 & 151 & 100 & 0 & 151 & 100 \\
\hline
\end{tabular}

The S/CO value of 2.721 in Tp0693-ELISA that maximizes expression sensitivity + specificity -1 was used as a cut-off value. PW, pregnant women; PE, physical examination (healthy patients); EBV, Epstein-Barr virus infection; Sty, Salmonella typhimurium infection; RD, rheumatic disease; EHF, epidemic hemorrhagic fever; AN, all negative samples; TPPA, Tp Particle Agglutination; TRUST, Tolulized Red Unheated Serum test; Tp, Treponema pallidum.

Table III. Distribution of different specimens in the detection of the grey zone of Tp0693-specific ELISA.

\begin{tabular}{lcc}
\hline Sample type & Total $(\mathrm{n})$ & Grey area samples, $\mathrm{n}(\%)$ \\
\hline PS & 36 & $1(2.78)$ \\
SS & 41 & $0(0)$ \\
TS & 12 & $1(8.3)$ \\
LS & 75 & $9(12)$ \\
CS & 4 & $0(0)$ \\
PW & 37 & $3(8.1)$ \\
PE & 51 & $12(23.5)$ \\
EBV & 22 & $17(77.3)$ \\
Sty & 10 & $8(80)$ \\
RD & 29 & $16(55.2)$ \\
ECH & 3 & $0(0)$ \\
MM & 1 & $0(0)$ \\
CMV & 1 & $1(100)$ \\
All & 321 & $68(21.2)$ \\
\hline
\end{tabular}

PS, primary syphilis; SS, secondary syphilis; TS, tertiary syphilis; LS, latent syphilis; CS, congenital syphilis; PW, pregnant women; PE, physical examination (healthy patients); EBV, Epstein-Barr virus infection; Sty, Salmonella typhimurium infection; RD, rheumatic disease; EHF, epidemic hemorrhagic fever; MM, multiple myeloma; $\mathrm{CMV}$, cytomegalovirus infection; All, all samples.

is low. Membrane protein TP47 and human as well as other treponema species (such as treponema endemicum and treponema pertenue) share high antigenic homology (16), leading to false-positive reactions. Therefore, it is necessary to explore novel types of recombinant antigens, and multi-epitope chimeric antigens may improve the specificity and sensitivity of detection.

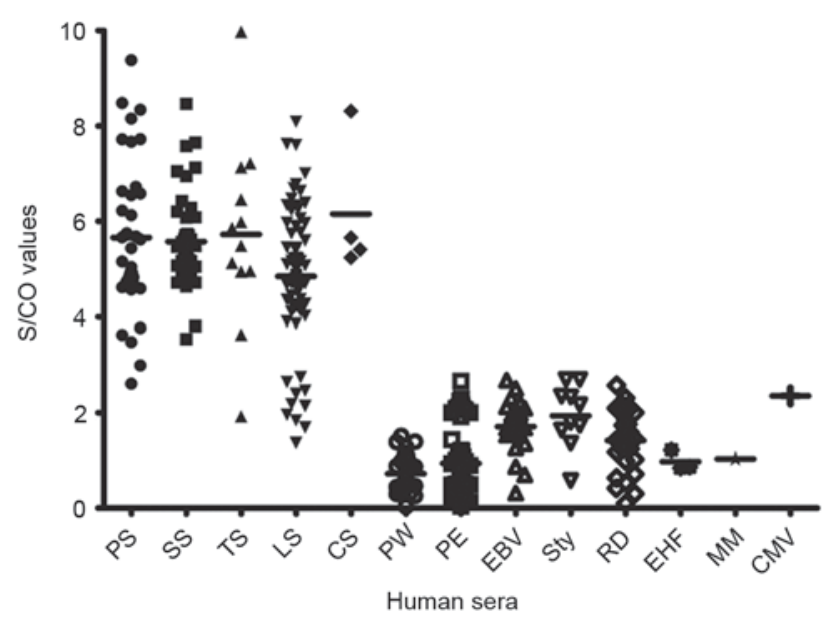

Figure 4. S/CO values for Tp0693-specific ELISA regarding the detection of different stages of syphilis and cross-reactivity in serum of patients without syphilis infection. The different stages of syphilis and other infections/diseases were grouped on the $\mathrm{x}$-axis and mean $\mathrm{S} / \mathrm{CO}$ values of each group were displayed on the $\mathrm{y}$-axis. The overall mean $\mathrm{S} / \mathrm{CO}$ value of each group is represented by horizontal lines. PS, primary syphilis; SS, secondary syphilis; TS, tertiary syphilis; LS, latent syphilis; CS, congenital syphilis; PW, pregnant women; PE, physical examination (healthy patients); EBV, Epstein-Barr virus infection; Sty, Salmonella typhimurium infection; $\mathrm{RD}$, rheumatic disease; EHF, epidemic hemorrhagic fever; MM, multiple myeloma; CMV, cytomegalovirus infection; S/CO, sample-to-cutoff ratio.

The structure and function of Tp0693 in Tp have remained elusive. Certain exocrine signaling peptides are present on the outer membrane of pathogens, and Tp0693 protein is stably expressed in the pathogenic strains of Tp according to a bioinformatics analysis. It shares low homology with other pathogens and the human genome. While McKevitt M (18) did not observe natural Tp0693 by protein mass spectrometric analysis of Tp bacterial protein, this may not necessarily indicate weak antigenicity and immunity; it may have been due to Tp0693 being an exocrine protein that is secreted by $\mathrm{Tp}$ 
A

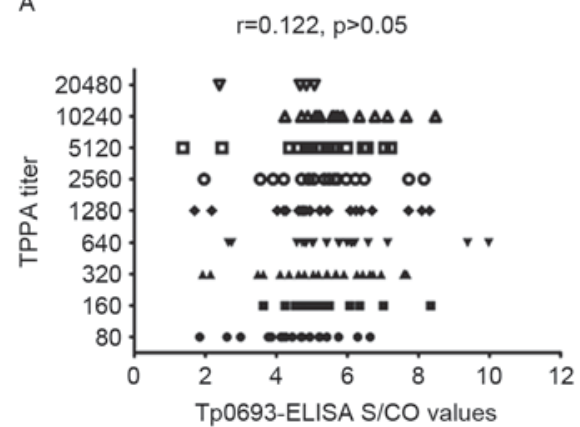

B

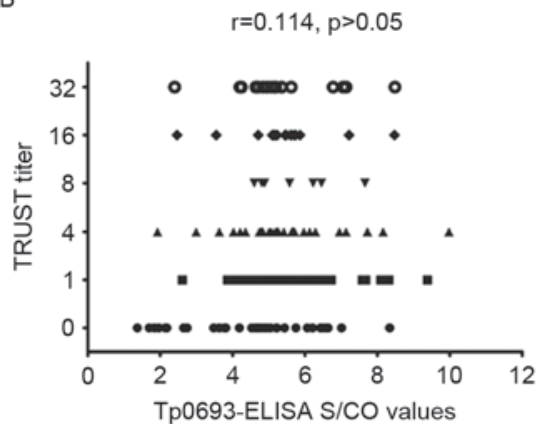

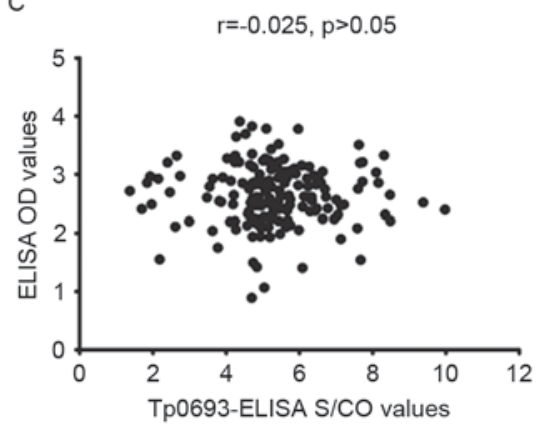

Figure 5. Correlation analysis of TPPA, TRUST, LiZhu ${ }^{\mathrm{TM}}$ Tp-ELISA and Tp0693-specific ELISA. (A) Correlation between the S/CO value for Tp0693-ELISA and the TPPA titer. (B) Correlation between the S/CO value for Tp0693-ELISA and the TRUST titer. (C) Correlation between the S/CO value for Tp0693-ELISA and OD values from LiZhu ${ }^{\mathrm{TM}}$ Tp-ELISA. S/CO, sample-to-cutoff ratio; TPPA, Tp Particle Agglutination; TRUST, Tolulized Red Unheated Serum test; OD, optical density; Tp, Treponema pallidum.

A

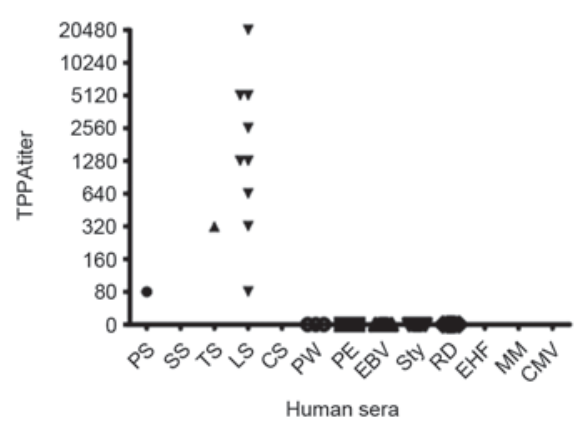

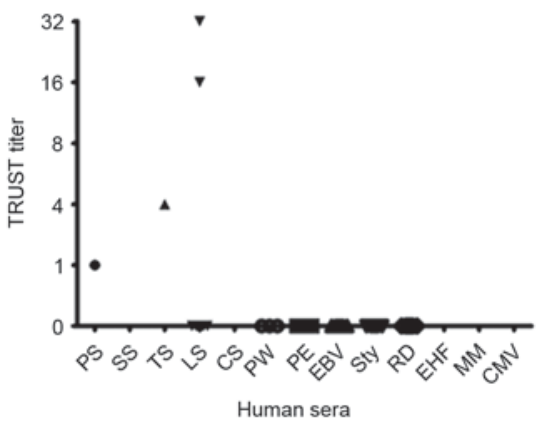

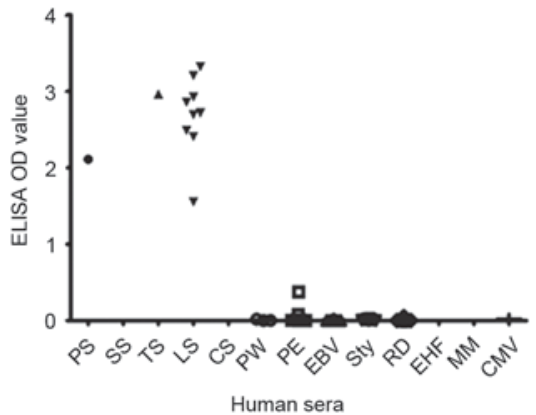

Figure 6. Grey area of Tp0693-specific ELISA. (A) TPPA titers, (B) TRUST titers and (C) ELISA S/CO values in serum of patient groups with different stages of syphilis and those without syphilis infection. PS, primary syphilis; SS, secondary syphilis; TS, tertiary syphilis; LS, latent syphilis; CS, congenital syphilis; PW, pregnant women; PE, physical examination (healthy patients); EBV, Epstein-Barr virus infection; Sty, Salmonella typhimurium infection; RD, rheumatic disease; EHF, epidemic hemorrhagic fever; MM, multiple myeloma; CMV, cytomegalovirus infection; S/CO, sample-to-cutoff ratio; TPPA, Tp Particle Agglutination; TRUST, Tolulized Red Unheated Serum test; OD, optical density; Tp, Treponema pallidum.

during the early stages of infection. Other pathogen-associated secreted proteins have been reported in clinical studies (26).

The present study successfully constructed pET30a/Tp0693 recombinant plasmid and purified Tp0693 recombinant protein. This purified protein had no interaction with serum from individuals without Tp infection, but strong immunoreactivity with the serum of patients with syphilis, indicating the antigen-specific reactions and potential diagnostic value of Tp0693.

The ROC curve indicated a correlation between sensitivity and specificity, with the false-positive rate (1-specificity) displayed on the $y$-axis and the true positive rate (sensitivity) on the $\mathrm{x}$-axis as. The AUC was used to evaluate the diagnostic value. There is no diagnostic value when the AUC is $<0.5$, a low diagnostic value when the AUC is $<0.7$, a moderate diagnostic value when the AUC is 0.7-0.9, and a significant diagnostic value when the AUC is $>0.9$. The AUC of the S/CO value from the TP0693-ELISA assay was 0.99 , indicating a high diagnostic value.

In the present study, the diagnostic specificity of Tp0693-ELISA reached $100 \%$ and the sensitivity was up to $93.5 \%$, which was higher than that of TRUST (79.2\%). The sensitivity of Tp0693-ELISA was $97.2 \%$ for primary syphilis. In the present study, the specificity of TPPA and TRUST was $100 \%$ each, which was higher than that determined by
Liu et al (27) (98.38\% for TPPA and $86.49 \%$ for TRUST). This discrepancy may be due to the limited number of collected samples and potential cross-reactions in the present study, for which the specificity of Tp0693-ELISA (100\%) was higher than that of LiZhu ${ }^{\mathrm{TM}}$ Tp-ELISA (88.9\%). However, in the clinical setting, the sensitivity of screening tests is more important than the specificity to avoid misdiagnosis (28), which may be avoided by another specific syphilis test. Therefore, it is acceptable that in the present study, the sensitivity of the ELISA screening test was lower.

In the syphilis-positive samples, the S/CO value of the Tp0693-ELISA was not correlated with the TPPA titer, RPR titer or OD value of the LiZhu ${ }^{\mathrm{TM}}$ Tp-ELISA. The TPPA titer was weakly correlated with the OD value of the $\mathrm{LiZhu}^{\mathrm{TM}}$ Tp-ELISA, which uses Tp membrane lipoprotein as the analyte antigen (data not shown). Previously, Yoshioka et al (29) reported that chemiluminescent microparticle immunoassay (CMIA) results were strongly correlated with TPPA titers. Jiang et al (24) reported that membrane protein TpF1-specific ELISA was correlated with TPPA titers. As TPPA assesses Tp antigens as a whole, i.e. all membrane lipoproteins combined, as antigens, its results are likely to be associated with lipoprotein, as well as the results of membrane antigen Tp-ELISA, CMIA and TpF1-ELISA. However, the Tp0693 protein is a completely different type of secretion protein 
whose levels may not be correlated with the results of other tests.

The gray area analysis missed a proportion of the serum samples from patients with syphilis, including those with high LiZhu ${ }^{\mathrm{TM}}$ Tp-ELISA OD values and high TPPA and RPR titers, which may be due to the low or no production of Tp0693 during certain periods of Tp infection. Part of the S/CO values of serum samples from syphilis-negative patients were in the grey area, which may be due to the Tp0693 protein having weak cross-reactivity with human proteins (30). In the future, epitope fitting of Tp0693 and other strong and specific antigens will be assessed as diagnostic antigens to improve the specificity and sensitivity of detection. Those samples in the grey area of Tp0693-ELISA should further perform TPPA for diagnosis instead of TRUST and ELISA. The latter two methods were not able to distinguish between true positives and true negatives.

In the present study, the $\mathrm{Tp}$ IgG antibody was detected, which is not suitable for detecting early syphilis and evaluating treatment efficacy, relapse and infectiousness. Bosshard (31) found that patients in the early infection stage may have negative TPPA results but may be positive for Tp IgM detected by ELISA. Due to its high molecular weight, the $\operatorname{Tp} \operatorname{IgM}$ antibody is not able to get through the placenta and blood brain barrier, and therefore, the identification of a specific Tp IgM antibody in cerebrospinal fluid and amniotic fluid may be of great importance for the diagnosis of congenital syphilis and neurosyphilis (32). Tp0693 is considered as a secretion protein that is only secreted during active infection and therefore, it may be better for diagnosing active infections. There are similar ongoing studies on the diagnostic potential of secretion proteins of Mycobacterium tuberculosis for active infection (26). Therefore, it is worthwhile to further analyze $\operatorname{Tp} \operatorname{IgM}$ antibody as an early sign of syphilis infection.

In conclusion, the present study evaluated the diagnostic performance of a novel secretion protein, Tp0693, in various stages of syphilis. Taken together, the results of the present study indicate that Tp0693 may be a promising antigenic marker of syphilis that can be developed into a diagnostic antigen for the screening of syphilis.

\section{Acknowledgements}

The present study was supported by the National Natural Science Foundation (grant nos. 81373230 and 81301470), the Guangdong Provincial Science and Technology Department Foundation (grant no. 2014A020212036), the Preclinical Medicine Hunan Provincial Key Disciplines, Hunan Provincial Key Laboratory for Special Pathogens Prevention and Control (grant no. 2014-5), the Hunan Provincial Cooperative Innovation Center for Molecular Target New Drug Study (grant no. 2015-08).

\section{References}

1. Chadwick JA, MacNab A, Sarma J, Ray S, Kadir I and Muldoon EG: Secondary syphilis presenting with aortitis and coronary ostial occlusion. Sex Transm Infect 92: 108-109, 2016.

2. Sukthankar A: Syphilis. Medicine 42: 394-398, 2014.
3. Stamm LV: Syphilis: Re-emergence of an old foe. Microb Cell 3: 363-370, 2016.

4. World Health Organization: Prevalence and incidence of selected sexually transmitted infections. Chlamydia trachomatis, Neisseria gonorrhoeae, syphilis and Trichomonas vaginalis: Methods and results used by WHO to generate 2005 estimates. World Health Organization, Geneva, 2011.

5. World Health Organization: Global prevalence and incidence of selected curable sexually transmitted infections: Overview and estimates. World Health Organization, Geneva, 2001.

6. Tucker JD, Chen XS and Peeling RW: Syphilis and social upheaval in China. N Engl J Med 362: 1658-1661, 2010.

7. Tucker JD and Cohen MS: China's syphilis epidemic: Epidemiology, proximate determinants of spread, and control responses. Curr Opin Infect Dis 24: 50-55, 2011.

8. Klein J, McLaud M and Rogers D: Syphilis on the Rise: Diagnosis, treatment and prevention. J Nurse Pract 11: 49-55, 2015.

9. Zanto SN: Changing algorithms in syphilis laboratory diagnosis. Clin Microbiol Newsl 32: 59-64, 2010.

10. Workowski KA and Bolan GA; Centers for Disease Control and Prevention: Sexually transmitted diseases treatment guidelines, 2015. MMWR Recomm Rep 64: 1-137, 2015.

11. Morales-Múnera CE, Fuentes-Finkelstein PA and Vall Mayans M: Update on the diagnosis and treatment of RR-Sífilis: Actualización en el manejo diagnóstico y terapéutico 106: 68-69, 2015.

12. Liu F, Liu LL, Guo XJ, Xi Y, Lin LR, Zhang HL, Huang SJ, Chen YY,Zhang YF,Zhang Q, et al: Characterization of the classical biological false-positive reaction in the serological test for syphilis in the modern era. Int Immunopharmacol 20: 331-336, 2014.

13. Eickhoff CA and Decker CF: Syphilis. Dis Mon 62: 280-286, 2016.

14. Sun AH, Mao YF, Hu Y, Sun Q and Yan J: Sensitive and specific ELISA coated by TpN15-TpN17-TpN47 fusion protein for detection of antibodies to Treponema pallidum. Clin Chem Lab Med 47: 321-326, 2009.

15. Brito Moreno AI, Acosta Bas C, Rodríguez M, Baluja Conde IB, Feal Carballo $\mathrm{S}$ and Martínez L: Monoclonal antibodies to the recombinant protein TmpA of the Treponema pallidum. Hybrid Hybridomics 22: 393-396, 2003.

16. Baughn RE, Jiang A, Abraham R, Ottmers V and Musher DM: Molecular mimicry between an immunodominant amino acid motif on the 47-kDa lipoprotein of Treponema pallidum (Tpp47) and multiple repeats of analogous sequences in fibronectin. J Immunol 157: 720-731, 1996.

17. Lafond RE and Lukeart SA: Biological basis for syphilis. Clin Microbiol Rev 19: 29-49, 2006.

18. McKevitt M, Brinkman MB, McLoughlin M, Perez C, Howell JK, Weinstock GM, Norris SJ and Palzkill T: Genome scale identification of Treponema pallidum antigens. Infect Immun 73: 4445-4450, 2005.

19. Pinedo-Cancino V, Laurenti MD, Kesper N and Umezawa ES: Evaluation of Leishmania (Leishmania) infantum excreted-secretion antigens for detection of canine leishmaniasis. Acta Trop 161: 41-43, 2016.

20. Rizvi N, Singh A, Yadav M, Hussain SR, Siddiqui S, Kumar V, Ali S and Agarwal A: Role of alpha-crystallin, early-secretion antigenic target $6-\mathrm{kDa}$ protein and culture filtrate protein 10 as novel diagnostic markers in osteoarticular tuberculosis. J Ortho Trans 6: 18-26, 2016.

21. Sabry MA, Taher ES, Allah NF and Mahgoub AM: Diagnosis of Fasciola infection by SDS-PAGE eluted excretory secretory (ES) protein fractions using dot-ELISA. Int J Vet Sci Med 2: 130-135, 2014.

22. Sánchez-Andrade R, Cortiñas FJ, Francisco I, Sánchez JA, Mula P, Cazapal C, Vázquez L, Suárez JL, Francisco R, Arias MS, et al: A novel second instar Gasterophilus excretory/secretory antigen-based ELISA for the diagnosis of gasterophilosis in grazing horses. Vet Parasitol 171: 314-320, 2010.

23. Xu M, Xie Y, Jiang C, Xiao Y, Kuang X, Zhao F, Zeng T, Liu S, Liang M, Li L, et al: A novel ELISA using a recombinant outer membrane protein, rTp0663, as the antigen for serological diagnosis of syphilis. Int J Infect Dis 43: 51-57, 2016.

24. Jiang C, Zhao F, Xiao J, Zeng T, Yu J, Ma X, Wu H and $\mathrm{Wu} \mathrm{Y}$ : Evaluation of the recombinant protein $\mathrm{TpF} 1$ of Treponema pallidum for serodiagnosis of syphilis. Clin Vaccine Immunol 20: 1563-1568, 2013.

25. Jiang C, Xiao J, Xie Y, Xiao Y, Wang C, Kuang X, Xu M, Li R, Zeng T, Liu S, et al: Evaluation of FlaB1, FlaB2, FlaB3, and Tp0463 of Treponema pallidum for serodiagnosis of syphilis. Diagn Microbiol Infect Dis 84: 105-111, 2016. 
26. Tiwari D, Haque S, Tiwari RP, Jawed A, Govender T and Kruger HG: Fast and efficient detection of tuberculosis antigens using liposome encapsulated secretory proteins of Mycobacterium tuberculosis. J Microbiol Immunol Infect 50: 189-198, 2017.

27. Liu C, Ou Q, Chen H, Chen J, Lin S, Jiang L and Yang B The diagnostic value and performance evaluation of five serological tests for the detection of Treponema pallidum. J Clin Lab Anal 28: 204-209, 2014.

28. Cantor AG, Pappas M, Daeges M and Nelson HD: Screening for Syphilis: Updated evidence report and systematic review for the US preventive services task force. JAMA 315: 2328-2337, 2016.

29. Yoshioka N, Deguchi M, Kagita M, Kita M, Watanabe M, Asari S and Iwatani Y: Evaluation of a chemiluminescent microparticle immunoassay for determination of Treponema pallidum antibodies. Clin Lab 53: 597-603, 2007.
30. Wang L, Deng X, Liu H, Zhao L, You X, Dai P, Wan K and Zeng Y: The mimic epitopes of Mycobacterium tuberculosis screened by phage display peptide library have serodiagnositic potential for tuberculosis. Pathog Dis 74: pii: ftw091, 2016.

31. Bosshard PP: Usefulness of IgM-specific enzyme immunoassays for serodiagnosis of syphilis: Comparative evaluation of three different assays. J Infect 67: 35-42, 2013.

32. Herremans M, Notermans DW, Mommers M and Kortbeek LM: Comparison of a Treponema pallidum IgM immunoblot with a 19S fluorescent treponemal antibody absorption test for the diagnosis of congenital syphilis. Diagn Microbiol Infect Dis 59: 61-66, 2007. 